\title{
Changes in Muscle Activity among College Pitchers Using 5- to 12-Oz Weighted Baseball
}

\author{
Won-Ho Choi' ${ }^{1}$ Yun-A Shin ${ }^{2 *}$ \\ ${ }^{1}$ Department of Physical Education, Graduate School, Dankook University \\ ${ }^{2}$ Department of Prescription \& Rehabilitation of Exercise, College of Physical Exercise, Dankook University
}

\begin{abstract}
Received: May 26, 2020

Accepted: October 15, 2020

Published online: October 31, 2020

Keywords:

College Pitcher

Joint Muscle

Muscle Activity

Rotator Cuff Muscle

Weighted Baseball

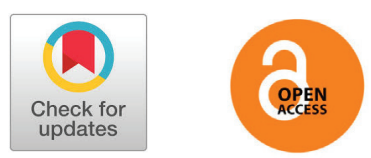

OBJECTIVES Several studies have reported that weighted baseball (WB) training is effective in improving ball speed; however, the weight of the ball suitable for training remains unclear. This study aimed to investigate the changes in muscle activity during pitching using 5- to12-oz WBs and to provide basic data for training programs to improve pitching speed.

METHODS The subjects of this study were 10 overhand pitchers who had more than 5 years of experience. Muscle activity was measured and analyzed at $70-85 \%$ of throwing baseball maximum effort (TBME) during soft toss (ST) and TBME was evaluated using electromyography.

RESULTS As the ball weight increased, muscle activity also increased in all pitching phases. Muscle activity was higher during ST with WBs heavier than 10 or $11 \mathrm{oz}$ than during TBME, indicating that the loads on the shoulder and elbow joint muscles increased. Conversely, muscle activity during ST with 5- to 7-oz WBs was lower than that during TBME, although phase and muscle group differences were observed.

CONCLUSIONS The results of this study suggest that training with 8- to 10-oz WBs could increase muscle strength and activity, although the effect may vary with fitness level and muscle strength.
\end{abstract}

(c) The Asian Society of Kinesiology and the Korean Academy of Kinesiology

\section{Introduction}

Overhead throwing is a common cause of injury among baseball players [1]. Throwing causes great stress on the upper extremities, and pain related to throwing is extremely common among baseball pitchers [2,3]. This is because pitching requires the upper extremities to generate substantial force and torque [4]. Consequently, the prevalence of shoulder and elbow pain among pitchers is high (46-57\%) [5]. Pitching speed of amateur and professional baseball players has been recently highlighted as an increase in pitching speed increases the risk

*Correspondence: Yun-A Shin, Department of Prescription \& Rehabilitation of Exercise, College of Physical Exercise, Dankook University, San 29, Anseo-dong, Cheonan-si, Chungnam 330-714, Republic of Korea; Tel: +82-41-550-3831; Fax: +8241-550-3831; E-mail: shinagel3@gmail.com of shoulder and elbow stress and damage [6-8]. Hence, proper training methods that could increase pitching speed while preventing injury among athletes are needed.

The throwing motion is a complex movement that requires muscle strength, coordination, flexibility, and neuromuscular efficiency, such as synchronicity of muscle firing [9]. Thus, an athlete's management and rehabilitation programs generally consist of activity modification, strengthening exercise, and flexibility exercise [10]. Among the various training programs, training with light balls (LBs) and weighted baseballs (WBs) has been reported to increase pitching speed and muscle strength during pitching [11-14]. Fleisig et al.[15] examined movement changes after throwing a regular ball (RB; $5 \mathrm{oz}$ ) and an LB (4 oz) among young pitchers (11.1 \pm 0.7 years) 
and reported that the shoulder and elbow speeds of external rotation and the ball speeds increased when an LB was used without change in arm position. Yang et al.[16] also reported that throwing speed and arm swing speed significantly increased after 10 weeks of training using 4.4- to 5-oz baseballs among adolescents, and they suggested that light WB training is effective in young players.

The ability to pitch is influenced by a pitcher's particular physical conditions; thus, the weight of the ball used for training must vary with age. Previous studies have suggested that WB training is more effective in increasing ball speed than RB training among high school and college baseball pitchers [11-14]. However, DeRenne et al. [17,18] conducted pitching training with 4- to 6-oz baseballs for 10 weeks in high school pitchers and found no difference between LB and WB training in increasing pitching speed. In addition, Escamilla et al. [13] reported a $3.20 \%$ increase in pitching speed after a 10-week training session with an LB weighing 4 to $4.72 \mathrm{oz}$ and a heavy ball weighing 5.25 to $17 \mathrm{oz}$. Therefore, inconsistent results have been reported, and the most appropriate ball weight for speed training remains unclear.

Recently, Fleisig et al. [19] measured ball speed and analyzed motion during pitching with balls weighing 4 to $32 \mathrm{oz}$. They found that the velocity of shoulder internal rotation, elbow varus torque, and ball speed increased when LBs were used; moreover, as the ball weight increased, the arm force, torque, and velocity decreased. However, ballistic training with WBs, an exercise that increases biceps muscle strength, has been reported to significantly decrease elbow torque but increase elbow flexion torque. $[19,20]$. The discrepancy in these results could be attributed to the use of motion analyses to investigate the differences in joint angular velocity while throwing WBs and whether this training increases pitching speed in several studies and to the analysis of muscle activity in relation to the generation of force during pitching a limited number of studies [20-23].
Surface electromyography (EMG) is used to analyze the pattern of the main muscle during the throwing phase. By analyzing EMG, information on the relationship between anatomical movement and temporal aspects of body movement, force generation, and fatigue could be obtained [24]. In particular, if the training can be performed to increase the main muscle strength in the pitching practice, it is considered to be helpful in the performance of the activity modification, throwing activities, coordination, and prevention of injury rather than separate muscle strength training.

Studies have used WBs of various weights for training; thus, the weight of the ball suitable for training is unclear. To identify the appropriate WB for baseball training, determining how ball weight could affect muscle mobilization and activity in pitchers is necessary. Thus, this study aimed to investigate muscle mobilization and activity during pitching using 5 - to 12-oz WBs and provide practical data for training programs to improve pitching motion and prevent injury.

\section{Methods}

\section{Subjects}

The study population consisted of 10 pitchers from "D" College in South Chungcheong Province. All pitchers were first-and second-year students with at least 5 years of pitching experience. All subjects were overhand pitchers and had no history of elbow or shoulder injury. The physical characteristics of the subjects are shown in $<$ Table $1>$.

\section{Electromyography (EMG) Electrode Attachment}

Muscle activation during the pitching test was measured using a wireless TELEmyo ${ }^{\mathrm{TM}} 2400$ EMG (Noraxon, Scottsdale, Arizona, USA). The skin was cleaned using alcohol to remove any foreign substances prior to the attachment of seven electrodes (distance between centers: $1.5 \mathrm{~cm}$ ). Subsequently, electrodes were attached to the rotator cuff muscles

Table 1. Subjects characteristics. Data are expressed as means \pm SD

\begin{tabular}{|c|c|c|c|c|c|c|}
\hline Position & $\mathbf{N}$ & Age (yrs.) & Height $(\mathrm{cm})$ & Weight (kg) & BMI $\left(\mathrm{kg} / \mathrm{m}^{2}\right)$ & Career (yrs.) \\
\hline Pitcher & 10 & $21.40 \pm 0.84$ & $178.0 \pm 2.71$ & $84.1 \pm 8.19$ & $26.56 \pm 2.57$ & $10.56 \pm 2.19$ \\
\hline
\end{tabular}


(supraspinatus, infraspinatus, and teres minor muscles) and elbow joint muscles (biceps brachii, triceps brachii, flexor carpi radialis, and extensor carpi radialis muscles) on the dominant side. Among the rotator cuff muscles, the subscapular was not measured, because EMG could not measure this muscle. Prior to the experiment, each subject had practice tests to ensure the measurement of maximal voluntary isometric contraction (MVIC) in each of the seven muscles. Each subject was provided enough rest prior to the actual measurement. The locations of the electrode attachment and MVIC measurements followed the guidelines presented in previous studies [25] and in the EMG manufacturer's protocol (SENIAM guidelines).

\section{MVIC Measurement}

To measure MVIC, the subjects performed specific motions for the aforementioned seven muscles for $5 \mathrm{~s}$. MVIC measurement was performed by lowering the arm and having the shoulder joint exert maximum force towards the ipsilateral ear (for the supraspinatus muscles, SPR), by exerting maximum force with the shoulder in external rotation (for the infraspinatus muscle, IFR), by standing in a straight line and exerting maximum force with the elbow extended to the side at $90^{\circ}$ (for the teres minor muscle, TM), with the elbow maintained at a $120^{\circ}$ angle while the forearm exerted maximum force towards the upper arm (for the biceps brachii muscle, BB), with the elbow maintained at a $90^{\circ}$ angle while the forearm exerted maximum force in the direction opposite to the upper arm (for the triceps brachii muscle, TB), and with the palm facing forward and the radiocarpal joint pointing approximately $30^{\circ}$ toward the back of the hand (for the flexor carpi radialis muscle, FCR; force was exerted with the palm of the hand). The MVIC of the extensor carpi radialis muscle (ECR) was measured in the opposite direction.

\section{Muscle Activity Measurement during Pitching}

Prior to pitching, subjects prepared for throw a baseball with maximum effort (TBME) by performing soft toss (ST) for 10-15 min. Long toss was defined as anything form 120 feet to 420 feet with arc or on a line, and soft toss was defined as anything less than long-toss [26]. RBs(5oz) and overweight balls (OBs;6 to 12oz) were used during ST, in which 10 pitches were performed at 70-85\% TBME speed. ST was performed at a rate of perceived exertion (RPE) was around $60 \sim 70 \%$ by loose and relaxed throwing with a large arc [27]. Thereafter, another 10 pitches were performed using RBs at 100\% TBME.

Muscle activation was measured during ST and TBME. The bandwidth of the EMG signals was filtered using a 10$\mathrm{Hz}$ high-pass filter and a $250-\mathrm{Hz}$ low-pass filter followed by full-wave rectification. Subjects were also recorded with 6-mm high-speed digital cameras while pitching. EMG data was synchronized with the video recordings and analyzed. Camera analysis was used to divide the motion during pitching into six phases. For muscle activation analysis, stride, arm-cocking, arm acceleration, and deceleration phases, in which rotator cuff muscles were highly recruited, were selected and analyzed [28].

\section{Statistical Analysis}

Data were analyzed using SPSS 18.0 for Windows (SPSS Inc., Chicago, IL, USA). All measured values are expressed as means and standard deviations. Repeated measures analysis of variance was used to analyze the changes in muscle activity according to baseball weight. The least significant difference method was used for post-hoc analysis. Statistical significance was set at $\mathrm{p}<.05$.

\section{Results}

\section{Changes in Muscle Activity with WBs in the Stride Phase}

Changes in muscle activity during TBME and ST using WBs in the stride phase are shown in <Table $2>$. As the ball weight increased, the muscle activity of SPR $(\mathrm{p}<.001)$, TM $(\mathrm{p}<.05), \mathrm{TB}(\mathrm{p}<.05)$, and FCR $(\mathrm{p}<.01)$ also increased. The post-test showed that the SPR had a higher muscle activity during ST of 7- to 8-oz ( $\mathrm{p}<.01)$, 9- to 10-oz ( $\mathrm{p}<.001)$, and 11to $12-\mathrm{oz}(\mathrm{p}<.01) \mathrm{WBs}$ and TBME $(\mathrm{p}<.01)$ than during ST of RBs. Muscle activity was higher during ST of 11- to 12-oz WBs than during TBME $(\mathrm{p}<.05)$. TM during ST of RBs showed a lower muscle activity than that during ST of 11- to 12-oz WBs $(\mathrm{p}<.05)$; TM during TBME showed a lower muscle activity than that during ST of $11-\mathrm{oz}$ WBs $(\mathrm{p}<.01)$. TB during ST of 


\begin{tabular}{|c|c|c|c|c|c|c|c|}
\hline $\begin{array}{l}\text { Ball Weight } \\
\text { (OZ) }\end{array}$ & $\begin{array}{l}\text { SPR } \\
\text { (uV, \%MVIC) }\end{array}$ & $\begin{array}{l}\text { IFR (uV, } \\
\text { \%MVIC) }\end{array}$ & $\begin{array}{l}\text { TM } \\
\text { (uV, \%MVIC) }\end{array}$ & $\begin{array}{l}\text { BB (uV, } \\
\% M V I C)\end{array}$ & $\begin{array}{l}\text { TB } \\
\text { (uV, \%MVIC) }\end{array}$ & $\begin{array}{l}\text { FCR } \\
\text { (uV, \%MVIC) }\end{array}$ & $\begin{array}{l}\text { ECR } \\
\text { (uV, \%MVIC) }\end{array}$ \\
\hline 5 & $103.68 \pm 24.05$ & $58.01 \pm 18.66$ & $50.07 \pm 15.39$ & $30.53 \pm 12.15$ & $34.14 \pm 16.27^{b^{*}}$ & $33.06 \pm 15.71^{b^{*}}$ & $37.65 \pm 19.83$ \\
\hline 6 & $106.09 \pm 32.39$ & $65.25 \pm 23.91$ & $52.17 \pm 17.49$ & $29.09 \pm 8.36$ & $36.29 \pm 20.78^{\mathrm{b}^{*}}$ & $33.80 \pm 14.70^{\mathrm{b}^{*}}$ & $34.04 \pm 13.20$ \\
\hline 7 & $124.16 \pm 27.45^{\mathrm{a}^{* *}}$ & $63.28 \pm 27.63$ & $51.86 \pm 13.94$ & $28.30 \pm 11.76$ & $46.58 \pm 27.52$ & $34.30 \pm 16.73^{b^{*}}$ & $40.34 \pm 15.41$ \\
\hline 8 & $144.91 \pm 28.07^{\mathrm{a}^{* *}}$ & $64.53 \pm 27.30$ & $51.12 \pm 16.26$ & $24.17 \pm 8.41$ & $42.76 \pm 25.21$ & $42.07 \pm 18.61$ & $45.38 \pm 12.77$ \\
\hline 9 & $142.85 \pm 27.71^{a^{a * *}}$ & $63.92 \pm 33.40$ & $53.77 \pm 19.77$ & $34.42 \pm 14.23$ & $37.13 \pm 18.68^{b^{*}}$ & $42.95 \pm 20.38$ & $62.29 \pm 58.15$ \\
\hline 10 & $141.92 \pm 38.21^{1^{a * * *}}$ & $69.58 \pm 27.97$ & $53.11 \pm 15.71$ & $26.17 \pm 13.59$ & $43.29 \pm 20.56$ & $45.65 \pm 20.77$ & $40.92 \pm 15.73$ \\
\hline 11 & $181.22 \pm 42.87^{a^{* *}, b^{*}}$ & $72.00 \pm 48.29$ & $61.55 \pm 17.15^{a^{*}, b^{* *}}$ & $33.45 \pm 13.59$ & $50.94 \pm 27.92^{\mathrm{a}^{*}}$ & $51.56 \pm 18.27^{\mathrm{a}^{*}}$ & $54.22 \pm 27.05$ \\
\hline 12 & $177.73 \pm 43.52^{a^{* *}, b^{*}}$ & $64.43 \pm 49.12$ & $65.68 \pm 24.41^{\mathrm{a}^{*}}$ & $35.24 \pm 14.23$ & $52.34 \pm 36.26^{\mathrm{a}+}$ & $53.17 \pm 27.29^{\mathrm{a}+}$ & $40.89 \pm 11.56$ \\
\hline TBME & $133.32 \pm 24.86^{\mathrm{a**}}$ & $86.62 \pm 36.28$ & $51.69 \pm 16.21$ & $37.82 \pm 14.84$ & $52.98 \pm 21.11^{\mathrm{a}^{* *}}$ & $47.36 \pm 20.44 \mathrm{a}^{\mathrm{a}^{*}}$ & $50.31 \pm 29.97$ \\
\hline $\mathrm{F}$ & 10.722 & .515 & 2.887 & 1.885 & 2.842 & 3.528 & 1.461 \\
\hline Sig & .000 & .840 & .010 & .081 & .010 & .002 & .192 \\
\hline \multirow{2}{*}{ Post-hoc } & $5<7,8,9,10,11,12$, M.E & - & $5<11,12$ & - & $5<11,12$, M.E & $5<11,12$, M.E & $-s$ \\
\hline & $M . E<11,12$ & - & $M . E<11$ & - & M.E $>5,6,9$ & ME. $>5,6,7$ & - \\
\hline
\end{tabular}

${ }^{*} P<.05,{ }^{*} P<.01,{ }^{* * *} P<.001,+$ : trend of the difference significance

Abbreviation: SPR, supraspinatus; IFR, infraspinatus; TM, teres minor; BB, bicephalus brachii; $T B$, triceps brachii; FCR, flexor carpi radialis; ECR, extensor carpi radialis; TBME, throwing baseball maximum effort; $a$, difference between RBs ( $5 \mathrm{oz})$ and WBs (6 to $12 \mathrm{oz})$; $\mathrm{b}$, difference between TBME and RBs and WBs

RBs showed a lower muscle activity than that during ST of $11-\mathrm{oz}(\mathrm{p}<.05)$ and12-oz $(\mathrm{p}=.058) \mathrm{WBs}$ and TBME $(\mathrm{p}<.01)$; TB during TBME showed a higher muscle activity than that during ST of 5-, 6-, and 9-oz WBs $(\mathrm{p}<.05)$. FCR showed a lower muscle activity during ST of RBs than during ST of 11$\mathrm{oz}(\mathrm{p}<.05)$ and 12-oz $(\mathrm{p}=.055) \mathrm{WBs}$ and TBME $(\mathrm{p}<.05)$; FCR muscle during ST of 5- to7-oz WBs showed a lower muscle activity than that during TBME $(\mathrm{p}<.05)$.

\section{Changes in Muscle Activity with WBs in the Arm- Cocking Phase}

Changes in muscle activity during TBME and ST using WBs in the arm-cocking phase are shown in $<$ Table $3>$. As the ball weight increased, the muscle activity of SPR $(\mathrm{p}<.05)$, IFR $(\mathrm{p}<.05)$, TM $(\mathrm{p}<.05)$, FCR $(\mathrm{p}<.001)$, and ECR $(\mathrm{p}<.001)$ also increased. Post-test showed that the SPR muscle had a higher muscle activity during ST of $10-\mathrm{Oz}(\mathrm{p}=.058)$ and $12-\mathrm{oz}(\mathrm{p}<.05)$ WBs than during ST of RBs. IFR showed a higher muscle activity during TBME than during ST of 5-, $6-$, and $8-\mathrm{oz}(\mathrm{p}<.05)$ and 9-oz $(\mathrm{p}<.01)$ WBs. TM showed a higher muscle activity during TBME than during ST of 5-,6-, and $8-\mathrm{oz}$ WBs $(\mathrm{p}<.05)$. FCR showed a lower muscle activity during ST of RBs than during CB of 7- to 10-oz ( $\mathrm{p}<.05), 11-$
$\mathrm{Oz}(\mathrm{p}<.01)$, and 12-oz $(\mathrm{p}<.05)$ WBs and TBME $(\mathrm{p}<.01)$; FCR showed a higher muscle activity during TBME than during ST of 5- to6-oz $(\mathrm{p}<.01)$ and 7- and 12-oz $(\mathrm{p}<.05)$ WBs and a lower muscle activity during TBME than during ST of 11-oz WBs $(\mathrm{p}<.05)$. ECR showed a lower muscle activity during ST of RBs than during CB of 6- to 7-oz ( $\mathrm{p}<.05), 8$ - to 11-oz $(\mathrm{p}<.01)$, and 12-oz $(\mathrm{p}=.054) \mathrm{WBs}$ and TBME $(\mathrm{p}<.01)$; ECR showed a higher muscle activity during TBME than during ST of 5- to 7-oz $(\mathrm{p}<.05)$ WBs.

\section{Changes in Muscle Activity with WBs in the Acceleration Phase}

Changes in muscle activity during TBME and ST using WBs in the acceleration phase are shown in $<$ Table $4>$. As the ball weight increased, the muscle activity of $\operatorname{SPR}(\mathrm{p}<.001)$, $\operatorname{IFR}(\mathrm{p}<.01), \mathrm{TM}(\mathrm{p}<.01), \mathrm{BB}(\mathrm{p}<.01)$, FCR $(\mathrm{p}<.001)$, and ECR $(\mathrm{p}<.001)$ also increased. Post-test showed that the SPR muscle had a lower muscle activity during ST of RBs than during ST of $8-\mathrm{oz}(\mathrm{p}<.05)$ and 9- to $12-\mathrm{oz}(\mathrm{p}<.01)$ WBs and TBME $(\mathrm{p}<.05)$; SPR during TBME showed a lower muscle activity than that during ST of $9-\mathrm{oz}(\mathrm{p}=.059), 10-\mathrm{oz}(\mathrm{p}<.05), 11-\mathrm{oz}$ $(\mathrm{p}<.01)$, and 12-oz $(\mathrm{p}<.05)$ WBs. IFR during TBME showed a higher muscle activity than that during ST of 5-oz $(\mathrm{p}<.01)$ 
Table 3. Muscle activity during arm cocking phase. Data are expressed as means \pm SD

\begin{tabular}{|c|c|c|c|c|c|c|c|}
\hline $\begin{array}{l}\text { Ball Weight } \\
(\mathrm{OZ})\end{array}$ & $\begin{array}{l}\text { SPR } \\
(\mathrm{uV}, \% \mathrm{MVIC})\end{array}$ & $\begin{array}{l}\text { IFR } \\
\text { (uV, \%MVIC) }\end{array}$ & $\begin{array}{l}\text { TM } \\
\text { (uV, \%MVIC) }\end{array}$ & $\begin{array}{l}\text { BB (uV, } \\
\% M V I C)\end{array}$ & $\begin{array}{l}\text { TB } \\
\text { (uV, \%MVIC) }\end{array}$ & $\begin{array}{l}\text { FCR } \\
\text { (uV, \%MVIC) }\end{array}$ & $\begin{array}{l}\text { ECR } \\
\text { (uV, \%MVIC) }\end{array}$ \\
\hline 5 & $100.73 \pm 29.72$ & $67.10 \pm 17.19^{b^{* *}}$ & $52.73 \pm 17.23^{\mathrm{b}^{*}}$ & $43.55 \pm 12.98$ & $44.94 \pm 19.89$ & $35.92 \pm 12.96^{b^{* *}}$ & $44.20 \pm 13.24^{\mathrm{b}^{*}}$ \\
\hline 6 & $109.01 \pm 18.35$ & $72.40 \pm 26.91^{b^{*}}$ & $48.66 \pm 16.93^{b^{*}}$ & $42.03 \pm 9.86$ & $50.41 \pm 22.21$ & $33.86 \pm 9.83^{b^{* *}}$ & $51.11 \pm 13.17^{a^{*}, b^{*}}$ \\
\hline 7 & $110.01 \pm 27.80$ & $74.96 \pm 30.08$ & $54.93 \pm 22.21$ & $37.29 \pm 10.81$ & $53.59 \pm 25.73$ & $40.16 \pm 13.71^{a^{*}, b^{*}}$ & $52.96 \pm 13.64^{\mathrm{a}^{*}, \mathrm{~b}^{*}}$ \\
\hline 8 & $120.57 \pm 24.80$ & $65.66 \pm 27.49^{b^{*}}$ & $55.60 \pm 23.01^{b^{*}}$ & $43.91 \pm 11.31$ & $56.16 \pm 25.52$ & $43.17 \pm 16.47^{\mathrm{a}^{*}}$ & $55.66 \pm 12.03^{a^{* *}}$ \\
\hline 9 & $116.43 \pm 30.03$ & $71.74 \pm 19.35^{b^{* *}}$ & $58.53 \pm 27.03$ & $42.81 \pm 15.67$ & $58.28 \pm 32.77$ & $43.88 \pm 18.72^{\mathrm{a}^{*}}$ & $61.75 \pm 16.89^{a^{* *}}$ \\
\hline 10 & $128.18 \pm 32.72^{a+}$ & $84.48 \pm 27.29$ & $59.21 \pm 30.98$ & $47.12 \pm 17.58$ & $56.81 \pm 36.72$ & $42.78 \pm 10.99$ & $68.74 \pm 19.34^{\mathrm{a}^{* *}}$ \\
\hline 11 & $136.95 \pm 32.69$ & $85.79 \pm 27.47$ & $63.94 \pm 26.02$ & $49.67 \pm 12.30$ & $56.09 \pm 36.55$ & $51.31 \pm 17.51^{\mathrm{a}^{* *}}$ & $72.39 \pm 22.89^{a^{* *}}$ \\
\hline 12 & $141.05 \pm 39.25^{\mathrm{a}^{*}}$ & $92.07 \pm 38.13$ & $75.11 \pm 48.42$ & $54.17 \pm 18.93$ & $56.72 \pm 31.08$ & $49.76 \pm 17.82^{a^{*}, b^{*}}$ & $91.46 \pm 61.78^{a+}$ \\
\hline TBME & $115.31 \pm 30.94$ & $104.81 \pm 23.01^{a^{*}}$ & $68.69 \pm 22.01^{\mathrm{a}^{*}}$ & $50.82 \pm 13.63$ & $50.2818 .23 \pm$ & $43.63 \pm 14.54^{\mathrm{a}^{* *}}$ & $59.66 \pm 12.33^{a^{* *}}$ \\
\hline $\mathrm{F}$ & 2.306 & 2.420 & 2.638 & 2.041 & .606 & 5.283 & 4.403 \\
\hline Sig & .035 & .028 & .018 & .061 & .768 & .000 & .000 \\
\hline \multirow{2}{*}{ Post-hoc } & $5<10,12$ & $5<M . E$ & $5<M . E$ & - & $-s$ & $5<7,8,9,10,11,12$, M.E & $5<6,7,8,9,10,11$, M.E \\
\hline & - & M.E $>5,6,8,9$ & $M E>5,6,8$ & - & - & $M . E>5,6,7, M . E<11$ & M.E $>5,6,7$ \\
\hline
\end{tabular}

${ }^{*} P<.05,{ }^{*} P<.01,{ }^{* * *} P<.001,+$ : trend of the difference significance

Abbreviation: SPR, supraspinatus; IFR, infraspinatus; TM, teres minor; BB, bicephalus brachii; TB, triceps brachii; FCR, flexor carpi radialis; ECR, extensor carpi radialis; TBME, throwing baseball maximum effort; a, difference between RBs ( $5 \mathrm{oz})$ and WBs ( 6 to $12 \mathrm{oz})$; $b$, difference between TBME and RBs and WBs

Table 4. Muscle activity during acceleration phase. Data are expressed as means \pm SD

\begin{tabular}{|c|c|c|c|c|c|c|c|}
\hline $\begin{array}{l}\text { Ball Weight } \\
\text { (OZ) }\end{array}$ & $\begin{array}{l}\text { SPR } \\
\text { (uV, \%MVIC) }\end{array}$ & $\begin{array}{l}\text { IFR } \\
\text { (uV, \%MVIC) }\end{array}$ & $\begin{array}{l}\text { TM } \\
\text { (uV, \%MVIC) }\end{array}$ & $\begin{array}{l}\text { BB } \\
\text { (uV, \%MVIC) }\end{array}$ & $\begin{array}{l}\text { TB } \\
\text { (uV, \%MVIC) }\end{array}$ & $\begin{array}{l}\text { FCR } \\
\text { (uV, \%MVIC) }\end{array}$ & $\begin{array}{l}\text { ECR } \\
\text { (uV, \%MVIC) }\end{array}$ \\
\hline 5 & $108.43 \pm 28.70^{b^{*}}$ & $83.62 \pm 16.47^{b^{* *}}$ & $69.33 \pm 13.67^{b^{*}}$ & $48.20 \pm 15.46^{b^{* *}}$ & $46.62 \pm 16.17$ & $36.50 \pm 11.52^{b^{* *}}$ & $60.60 \pm 15.30^{b^{* *}}$ \\
\hline 6 & $121.42 \pm 34.53$ & $94.59 \pm 9.40^{\mathrm{b}^{*}}$ & $69.77 \pm 15.96^{b^{*}}$ & $43.81 \pm 16.21^{b^{* *}}$ & $56.65 \pm 22.93$ & $39.66 \pm 11.98^{\mathrm{b}^{*}}$ & $61.84 \pm 9.98^{\mathrm{b**}}$ \\
\hline 7 & $127.32 \pm 34.48$ & $95.28 \pm 5.69^{b^{*}}$ & $68.35 \pm 15.21^{b^{*}}$ & $45.26 \pm 20.81^{b^{*}}$ & $56.44 \pm 31.33$ & $41.94 \pm 14.17^{\mathrm{a}+}$ & $69.32 \pm 10.02^{\mathrm{a}^{*}}$ \\
\hline 8 & $150.22 \pm 51.68^{a^{*}}$ & $99.23 \pm 15.70^{\mathrm{b}^{*}}$ & $67.51 \pm 17.04^{b^{*}}$ & $56.57 \pm 17.51$ & $53.50 \pm 36.80$ & $46.23 \pm 16.24^{\mathrm{a}^{* *}}$ & $68.97 \pm 11.61$ \\
\hline 9 & $152.42 \pm 51.56^{\mathrm{a}^{* *} \mathrm{~b}+}$ & $94.72 \pm 24.19^{\mathrm{b}^{*}}$ & $71.29 \pm 13.35$ & $55.67 \pm 22.18$ & $53.07 \pm 23.19$ & $48.03 \pm 16.99^{\mathrm{a}^{* *}}$ & $74.54 \pm 16.14 \mathrm{a}^{\mathrm{a}^{*}}$ \\
\hline 10 & $156.04 \pm 29.98^{\mathrm{a}^{* *}, \mathrm{~b}^{*}}$ & $101.58 \pm 23.28$ & $74.93 \pm 16.47$ & $53.88 \pm 26.44$ & $56.35 \pm 32.32$ & $48.39 \pm 17.99^{\mathrm{a}^{* *}}$ & $79.38 \pm 18.44^{\mathrm{a}^{* * *}}$ \\
\hline 11 & $177.41 \pm 47.20^{\mathrm{a}^{* *}, \mathrm{~b}^{* *}}$ & $103.01 \pm 23.47^{b^{*}}$ & $77.40 \pm 24.40$ & $64.08 \pm 24.96^{\mathrm{a+}}$ & $63.09 \pm 39.31$ & $52.64 \pm 20.49^{\mathrm{a}^{* *}}$ & $86.06 \pm 23.07^{a^{* *}, b+}$ \\
\hline 12 & $168.43 \pm 49.13^{a^{* *}, b^{*}}$ & $108.20 \pm 33.44$ & $83.79 \pm 24.54^{a+}$ & $62.43 \pm 28.50$ & $69.62 \pm 49.97$ & $57.22 \pm 22.28^{\mathrm{a}^{* *}, \mathrm{~b}^{*}}$ & $100.72 \pm 44.48^{\mathrm{a}^{*}}$ \\
\hline TBME & $124.95 \pm 33.23^{a^{*}}$ & $131.68 \pm 30.05^{\mathrm{a}^{* *}}$ & $78.50 \pm 20.33^{a^{*}}$ & $62.03 \pm 16.66^{a^{*}}$ & $47.87 \pm 18.69$ & $46.01 \pm 13.85^{\mathrm{a}^{* *}}$ & $72.88 \pm 13.93^{\mathrm{a}^{* *}}$ \\
\hline $\mathrm{F}$ & 5.568 & 3.320 & 3.668 & 3.121 & 689 & 6.421 & 5.792 \\
\hline Sig & .000 & .004 & .002 & .005 & .700 & .000 & .000 \\
\hline \multirow{2}{*}{ Post-hoc } & $5<8,9,10,11,12$, M.E & $5<M . E$ & $5<12$, M.E & $5<11$, M.E & - & $5<7,8,9,10,11,12$, M.E & $5<7,8,9,10,11,12$, M.E \\
\hline & M.E $>5, M . E<10,11,12$ & M.E $>5,6,7,8,9,11$ & M.E $>5,6,7,8$ & $M . E>5,6,7$ & - & $M . E>5,6, M . E<12$ & M.E $>5,6,11$ \\
\hline
\end{tabular}

${ }^{*} P<.05,{ }^{* *} P<.01,{ }^{* * *} P<.001,+$ : trend of the difference significance

Abbreviation: SPR, supraspinatus; IFR, infraspinatus; TM, teres minor; BB, bicephalus brachii; TB, triceps brachii; FCR, flexor carpi radialis; ECR, extensor carpi radialis; TBME, throwing baseball maximum effort; $\mathrm{a}$, difference between RBs (5 oz) and WBs (6 to 12oz); b, difference between TBME and RBs and WBs

and 6- to 9-oz and11-oz ( $\mathrm{p}<.05)$ WBs. TM during ST of RBs showed a lower muscle activity than that during ST of $12-\mathrm{oz}$ $(\mathrm{p}=.059)$ WBs. TM during TBME showed a higher muscle activity than that during $\mathrm{CB}$ of 5 - to $8-\mathrm{oz}(\mathrm{p}<.05)$ WBs. BB during ST of RBs showed a lower muscle activity than that during ST of 11-oz ( $\mathrm{p}=.052)$ WBs. BB during TBME showed a higher muscle activity than that during ST of 5- to6-oz $(\mathrm{p}<.01)$ and7-oz (p<.05) WBs. FCR during ST of 5-oz WBs showed a lower muscle activity than that during ST of 7-oz $(\mathrm{p}=.054)$ and 8 - to $12-\mathrm{oz}(\mathrm{p}<.01) \mathrm{WBs}$ and TBME $(\mathrm{p}<.01)$. The muscle 
activity of FCR during TBME was higher than that during ST of $6-\mathrm{oz}$ WBs and lower than that during ST of $12-\mathrm{oz}(\mathrm{p}<.05)$ WBs. ECR during ST of RBs showed a lower muscle activity than that during ST of 7- to8-oz $(\mathrm{p}<.01), 9-\mathrm{oz}(\mathrm{p}<.05), 10-$ to11-oz $(\mathrm{p}<.01)$, and $12-\mathrm{oz}(\mathrm{p}<.05) \mathrm{WBs}$ and TBME $(\mathrm{p}<.01)$. The muscle activity of ECR during TBME was higher than that during ST of 5- to6-oz $(\mathrm{p}<.01)$ and 11-oz $(\mathrm{p}=.052)$ WBs.

\section{Changes in Muscle Activity with WBs in the Deceleration Phase}

Changes in muscle activity during TBME and ST using WBs in the deceleration phase are shown in $<$ Table $5>$. As the ball weight increased, muscle activity of $\mathrm{TM}(\mathrm{p}<.001)$, $\mathrm{BB}(\mathrm{p}<.05), \mathrm{TB}(\mathrm{p}<.001)$, FCR $(\mathrm{p}<.001)$, and ECR $(\mathrm{p}<.001)$ also increased. Post-test showed that TM had a lower muscle activity during ST of RBs than during ST of $12-\mathrm{oz}$ WBs and TBME ( $\mathrm{p}<.05)$; the muscle activity during TBME was higher than that during ST of 5- to 7-oz $(\mathrm{p}<.05)$ and $8-\mathrm{oz}(\mathrm{p}<.01)$ WBs. BB during TBME showed a higher muscle activity than that during ST of 5-oz $(\mathrm{p}<.01)$ and 6-oz $(\mathrm{p}<.05)$ WBs. TB during ST of RBs showed a lower muscle activity than that during ST of 7-oz (p<.05),8-oz (p<.01),9-oz (p<.05),10-oz (p<.01), and 11- to $12-\mathrm{oz}(\mathrm{p}<.05) \mathrm{WBs}$ and TBME $(\mathrm{p}<.05)$. FCR during ST
B of RBs showed a lower muscle activity than that during ST of 7-oz ( $\mathrm{p}<.01), 8-\mathrm{oz}(\mathrm{p}<.05)$, and 9- to 12-oz ( $\mathrm{p}<.01)$ WBs and TBME $(\mathrm{p}<.01)$; the muscle activity during TBME was lower than that during ST of $11-$ to $12-\mathrm{oz}(\mathrm{p}<.05)$ WBs. ECR during ST of RBs showed a lower muscle activity than that during ST of 6-, 8-, and 10-oz (p<.05) and 11- to12-oz (p<.01) WBs and TBME $(\mathrm{p}<.001)$; the muscle activity during TBME was higher than that during ST of 6- to7-oz $(\mathrm{p}<.05)$ WBs.

\section{Discussion}

Various programs to improve pitching motion are available. For amateur players, the most popular is training with underweight balls and WBs. This training program has been reported to enhance mechanics, arm speed, and arm strength and to increase pitching speed by $\geq 5 \mathrm{mph}(2.2 \mathrm{~m} / \mathrm{s})$ [29]. Although several studies have reported that WB training is effective in improving ball speed [11-14], arm velocity, trunk velocity, and arm force decrease as ball weight increases from 5 to $7 \mathrm{oz}$ [19]. In addition, Reinold et al.[29] reported mixed outcomes in pitchers training with 2-, 4-, 6-, 16-, and 32oz WBs three times a week. In that study, while a majority (80\%) of 19 players experienced an increase in ball speed, the

\begin{tabular}{|c|c|c|c|c|c|c|c|}
\hline $\begin{array}{l}\text { Ball Weight } \\
(\mathrm{OZ})\end{array}$ & $\begin{array}{l}\text { SPR } \\
\text { (uV, \%MVIC) }\end{array}$ & $\begin{array}{l}\text { IFR } \\
(\mathrm{uV}, \% \mathrm{MVIC})\end{array}$ & $\begin{array}{l}\text { TM } \\
\text { (uV, \%MVIC) }\end{array}$ & $\begin{array}{l}\text { BB } \\
\text { (uV, \%MVIC) }\end{array}$ & $\begin{array}{l}\text { TB } \\
\text { (uV, \%MVIC) }\end{array}$ & $\begin{array}{l}\text { FCR } \\
\text { (uV, \%MVIC) }\end{array}$ & $\begin{array}{l}\text { ECR } \\
(u V, \% M V I C)\end{array}$ \\
\hline 5 & $78.50 \pm 28.74$ & $54.83 \pm 20.89$ & $53.81 \pm 11.33^{b^{*}}$ & $30.97 \pm 12.51^{b^{* *}}$ & $26.56 \pm 12.71^{b^{*}}$ & $38.05 \pm 5.67^{b^{* *}}$ & $40.12 \pm 13.47^{b^{* * *}}$ \\
\hline 6 & $80.96 \pm 10.90$ & $51.55 \pm 27.53$ & $53.52 \pm 17.27^{\mathrm{b}^{*}}$ & $34.18 \pm 14.12^{\mathrm{b}^{*}}$ & $29.79 \pm 14.04$ & $40.43 \pm 6.99$ & $45.48 \pm 13.81^{\mathrm{a}^{*}, \mathrm{~b}^{*}}$ \\
\hline 7 & $98.21 \pm 45.20$ & $49.24 \pm 16.02$ & $62.40 \pm 12.77^{b^{*}}$ & $37.45 \pm 14.65$ & $34.86 \pm 18.64^{\mathrm{a}^{*}}$ & $45.04 \pm 9.20^{\mathrm{a}^{* *}}$ & $44.00 \pm 13.99^{b^{*}}$ \\
\hline 8 & $106.79 \pm 25.78$ & $56.46 \pm 30.25$ & $59.56 \pm 12.43^{b^{* *}}$ & $39.92 \pm 13.95$ & $42.04 \pm 17.92^{\mathrm{a}^{* *}}$ & $48.48 \pm 8.68^{a^{*}}$ & $52.70 \pm 17.66^{a^{*}}$ \\
\hline 9 & $99.30 \pm 60.45$ & $49.21 \pm 34.18$ & $65.68 \pm 14.87$ & $40.41 \pm 16.81$ & $51.17 \pm 26.93^{\mathrm{a}^{*}}$ & $47.17 \pm 7.02^{\mathrm{a}^{* *}}$ & $49.89 \pm 13.22$ \\
\hline 10 & $96.77 \pm 49.50$ & $73.61 \pm 39.33$ & $65.97 \pm 14.98$ & $43.14 \pm 18.04^{\mathrm{a}^{*}}$ & $49.29 \pm 18.94^{\mathrm{a}^{* *}}$ & $52.01 \pm 8.51^{\mathrm{a}^{* *}}$ & $56.94 \pm 17.75^{\mathrm{a}^{*}}$ \\
\hline 11 & $113.96 \pm 42.98$ & $98.13 \pm 101.20$ & $68.99 \pm 20.24$ & $45.78 \pm 20.27^{a^{*}}$ & $52.91 \pm 23.52^{\mathrm{a}^{*}}$ & $51.37 \pm 7.51^{\mathrm{a}^{* *}, \mathrm{~b}^{*}}$ & $64.18 \pm 14.02^{\mathrm{a}^{* *}}$ \\
\hline 12 & $124.46 \pm 50.36$ & $79.44 \pm 42.64$ & $87.05 \pm 26.14 \mathrm{a}^{\mathrm{a}^{*}}$ & $48.42 \pm 19.99^{a+}$ & $57.59 \pm 34.45^{a^{*}}$ & $57.81 \pm 9.32^{a^{* *}, b^{*}}$ & $63.53 \pm 18.10^{\mathrm{a}^{* *}}$ \\
\hline TBME & $80.67 \pm 22.62$ & $77.79 \pm 14.23$ & $79.43 \pm 14.66^{\mathrm{a}^{*}}$ & $44.96 \pm 11.79^{a^{* *}}$ & $43.39 \pm 21.02^{\mathrm{a}^{*}}$ & $44.10 \pm 6.08^{\mathrm{a}^{* *}}$ & $53.14 \pm 14.06^{\mathrm{a}^{* * *}}$ \\
\hline $\mathrm{F}$ & 1.445 & 1.362 & 4.662 & 2.762 & 4.803 & 12.642 & 5.047 \\
\hline Sig & .203 & .233 & .000 & .014 & .000 & .000 & .000 \\
\hline \multirow{2}{*}{ Post-hoc } & - & - & $5<12$, M.E & $5<10,11,12$, M.E & $5<7,8,9,10,11,12$, M.E & $5<8,9,10,11,12 . M . E$ & $5<6,8,10,11,12$, M.E \\
\hline & - & - & M.E. $>5,6,7,8$ & M.E $>5,6$ & M.E $>5$ & $M . E>5, M . E<11,12$ & $M . E<5,6,7$ \\
\hline
\end{tabular}

${ }^{*} P<.05,{ }^{*} P<.01,{ }^{* * *} P<.001,+$ : trend of the difference significance

Abbreviation: SPR, supraspinatus; IFR, infraspinatus; TM, teres minor; BB, bicephalus brachii; TB, triceps brachii; FCR, flexor carpi radialis; ECR, extensor carpi radialis; TBME, throwing baseball maximum effort; $a$, difference between RBs ( $5 \mathrm{Oz})$ and WBs ( 6 to $12 \mathrm{oz})$; $\mathrm{b}$, difference between TBME and RBs and WBs 
rest experienced no change or even a decrease in ball speed. Particularly, external rotation passive range of motion of the group trained with WBs increased by $4.3^{\circ}$, and $24 \%$ of the athletes reported increased injuries during training and subsequent seasons.

Moreover, elbow injury also appears most frequently among male baseball pitchers between 15 and 24 years old [30-32]. Young pitchers' elbow injuries have been linked to the joint stresses due to excessive valgus loading at the throwing elbow $[33,34]$. Thus, this study investigated changes in rotator cuff and forearm muscle activity as the weight of the ball was gradually increased in an attempt to determine the suitable weight for WBs during pitching.

In the stride phase, which is the initial phase, velocity is generated through linear forward movements and positioning of the arm in a cocking position, and elastic energy generated by the legs is transferred to the torso and the arm. At the initial point of contact with the ground, the abduction angle of the throwing shoulder is approximately $80-100^{\circ}$, and the deltoid and SPR are activated to maintain opening and abduction [22,35]. In addition, the shoulder of the throwing arm is horizontally abducted to the back of the trunk, and the posterior triceps, latissimus dorsi, TM, and posterior rotator cuff muscles are activated [23].

The increase in SPR activity seemed to maintain shoulder flexion [36,37] as the ball weight increased, while increased TM activity seemed to maintain horizontal abduction [36]. Increased TB activity as the ball weight increased seemed to maintain the extension of the arm in motion, and increased FCR activity appeared as a preparation for the next phase, i.e., from stride to arm-cocking phase. However, SPR activity was higher during TBME than during ST with RBs and was also higher during ST with 11-to 12-oz WBs than during TBME. Moreover, muscle activity of TM, TB, and FCR was also higher during TBME than during ST with 11-oz WBs.

The rotator cuff muscles play a role in preventing joint dislocations by holding the humeral head and the scapula together during shoulder movement [38]. As the ball weight increases, the load increases, thereby resulting in excessive SPR activation. Muscle balance problem in the posterior rotator cuff muscles is associated with humeral joint instability and impingement syndrome [39]. Moreover, training with a ball weighing $\geq 11 \mathrm{oz}$ without increased muscle strength may increase the risk of injury, as suggested by Reinold et al.[29]; thus, these WBs should only be used after sufficient training to strengthen muscles.

The arm-cocking phase, during which energy of the lower extremities and core is transferred and stored in the shoulder capsule as potential energy, begins with front foot strike and separation. The shoulders maintain $90^{\circ}$ abduction, and the glenohumeral and scapulothoracic joints rotate together up to $180^{\circ}$. In the late arm-cocking phase, shoulder rotational torque and valgus torque of the elbow are maximized; thus, this phase is significant in superior labral anterior posterior tears and ulnar collateral ligament (UCL) tears [40,41]. In this phase, the posterior triceps, latissimus dorsi, TM, and IFR are responsible for the external rotation of the shoulder [9], and wrist muscle activity is increased by cocking.

In our study, as the ball weight increased, muscle activity of SPR, FCR and ECR also increased. The increase in SPR activity was greater than that during ST with $10-$ and $12-\mathrm{oz}$ WBs, similar to the stride phase, because shoulder flexion was maintained. Furthermore, in the arm-cocking phase, FCR and ECR activity increased gradually as the ball weight increased. As most previous studies have primarily examined the activity of shoulder muscles, the extent of impairment of wrist muscle activity remains unclear. Nonetheless, flexor-pronator mass provides dynamic stability because of its anatomical location over the UCL [42]. Moreover, ball weights $>10 \mathrm{oz}$ may affect the wrist and elbow joints because ST of 10- to 12-oz WBs is associated with higher levels of elbow muscle activity, including FCR and ECR, compared with TBME.

The acceleration phase is where all the energy generated by the body is transferred to the ball. The explosive power of the internal rotators and the potential energy stored in the capsule are used during shoulder rotation. Moreover, the elbow is extended and the wrist is flexed, adding extra speed to the ball [43]. It has been reported that, during maximal external rotation, $111 \mathrm{~N} \mathrm{~m}$ of internal torque is generated as the pitcher moves from the cocking to the acceleration phase.30 Furthermore, the greatest muscle activity was observed in this phase [41]. 
Furthermore, in the acceleration phase, abduction of the upper arm bones, horizontal abduction, and internal rotation occur at a velocity of $7000 \mathrm{deg} / \mathrm{s}$ and a pressure of $800 \mathrm{~N}$ $[22,35]$. Shin and Choi $[21]$ reported that the activity of the upper trapezius, SPR, deltoid, latissimus dorsi, and TB is high during RB pitching and that IFR and serratus muscle activity is increased during WB pitching. In our study, IFR, TM, and TB activity increased as the ball weight increased but was not higher than that during TBME; thus, it could be considered a range that does not yield any strain to the muscle group. However, SPR and FCR showed a higher activity than that during ST of 10- and 12-oz WBs. This finding may be related to the increased muscle activity in the stride and arm-cocking phases.

IFR, TM, BB, FCR, and ECR activity during pitching using 5- to 7-oz WBs was significantly lower than that during TBME. This result possibly indicates that the WB (6 and $7 \mathrm{oz}$ ) did not stimulate muscle activity despite the increased weight to more than that of the $\mathrm{RB}$ and the pitching performed at $70-80 \%$ TBME. However, the result is inconsistent with that of previous studies, which reported that muscle strength increased after ballistic training with OBs [20] and that heavy ball holds are beneficial in increasing biceps muscle strength by increasing elbow flexion torque [19]. The inconsistent results between our study and previous studies could be attributed to the difference in the subjects investigated; in previous studies, WB training programs were evaluated in adolescents and high school students. The subjects in this study, on the other hand, were college students and differ from subjects in previous studies in physique, strength, and careers. Therefore, WBs that is heavier than those used during pitching in adolescents could be more effective in increasing muscle mobilization and activity during pitching in adults.

The deceleration phase begins when the arm slows down while releasing the ball. As the arm continues to adduct, the angular velocity decreases [44]. The proximal or compressive forces at the shoulder peak at several times of the body weight when the rotator cuff resists distractive momentum of the arm [45]. When releasing the ball, the elbow flexion angle is related to the kinetics of the shoulder and elbow, and minimal difference among pitchers was observed. Small differences could change the lever arm of the forearm and humerus. For example, elbow flexion could increase the length of the lever arm, the moment of inertia during humeral rotation, elbow valgus torque, as well as pressure on the UCL $[2,46]$.

$\mathrm{TM}$ and $\mathrm{BB}$ work to adduct and flex the shoulder and forearm, while TB works as an antagonist. As the weight of the ball increased, muscle activity also increased, which is similar to that during TBME. Therefore, the rotator cuff did not seem to be overloaded by weight in this phase. Moreover, muscle activity at the wrist joint increased as the ball weight increased. FCR activity was higher during 10- to $12-\mathrm{oz}$ WB pitching than during TBME. The acceleration phase is associated with the highest risk of injury; however, the deceleration phase therein is shifted within a very short time of $0.03 \mathrm{~s}$ and 0.04 $s$, respectively. With the excessive flexion of the FCR muscle, which increases the lever arm length and traction on the UCL [47], these results regarding the acceleration phase suggest load on the shoulder and elbow may be increased.

The limitation was that the number of subjects in this study was insufficient because only pitchers were recruited. Therefore, this study suggested that further study of more subjects is needed.

\section{Conclusions}

Our analysis on the muscle activity during pitching with WBs showed that the rotator cuff and elbow joint muscle activities increase as the ball weight increase. Muscle activity was higher during ST of 10- or 11-oz WBs than during TBME, and shoulder and elbow joint loads increased. By contrast, muscle activity during ST of 5- to 7-oz WBs was lower than that during TBME, although differences depending on the phase and muscle group were noted. Therefore, although training with WBs varies with a player's fitness level and muscle strength, our findings suggest that ball weights of 8 to $10 \mathrm{oz}$ can increase muscle strength and activity. These results may be practical when planning training with WBs and may help in preventing injury due to overuse and fatigue and in improving pitching speed. 


\section{Acknowledgments}

The authors are grateful to the baseball players who participated in this study and to the $\mathrm{D}$ university baseball coach Yoo-Jin Kim who assisted in the experiment.

\section{Conflicts of Interest}

The authors declare that there is no conflict of interests regarding the publication of this article.

\section{References}

1. Krajnik S, Fogarty KJ, Yard EE, Comstock RD. Shoulder injuries in US high school baseball and softball athletes, 2005-2008. Pediatrics. 2010; 125:497-501.

2. Aguinaldo AL, Chambers H. Correlation of throwing mechanics with elbow valgus load in adult baseball pitchers. Am J Sports Med. 2009; 37:2043-2048.

3. Aguinaldo AL, Buttermore J and Chambers H. Effects of upper trunk rotation on shoulder joint torque among baseball pitchers of various levels. J Appl Biomech. 2007; 23:42-51.

4. Werner SL, Gill TJ, Murray TA, Cook TD, Hawkins RJ. Relationships between throwing mechanics and shoulder distraction in professional baseball pitchers. Am J Sports Med. 2001; 29:354-358.

5. Lyman S, Fleisig GS, Andrews JR, Osinski ED. Effect of pitch type, pitch count, and pitching mechanics on risk of elbow and shoulder pain in youth baseball pitchers. Am J Sports Med. 2002; 30:463-468.

6. Bushnell BD, Anz AW, Noonan TJ, Torry MR, Hawkins RJ. Association of maximum pitch velocity and elbow injury in professional baseball pitchers. Am J Sports Med. 2010; 38:728-732.

7. Chalmers PN, Erickson BJ, Ball B, Romeo AA, Verma NN. Fastball pitch velocity helps predict ulnar collateral ligament reconstruction in major league baseball pitchers. Am J Sports Med. 2016; 44:2130-2135.

8. Hurd WJ, Jazayeri R, Mohr K, Limpisvasti O, Elattrache NS, Kaufman KR. Pitch velocity is a predictor of medial elbow distraction forces in the uninjured high school- aged baseball pitcher. Sports Health. 2012; 4:415-418.

9. Wilk KE, Meister K, Fleisig G, Andrews JR. Biomechanics of the overhead throwing motion. Sports Med Arthrosc Rev. 2000; 8:124-134.

10. Wilk KE, Meister K, Andrew JR. Current concepts in the rehabilitation of the overhead throwing athlete. Am J Sports Med. 2002; 3:136-151.

11. DeRenne C, Buxton BP, Hetzler RK, Ho KW. Effects of under and overweighed implement training on pitching velocity. J Strength Cond Res.1994; 8:247-250.

12. DeRenne C, Szymanski DJ. Effects of baseball weighted implement training: A brief review. J Strength Cond Res. 2009; 31:30-37.

13. Escamilla RF, Speer KP, Fleisig GS, Barrentine SW, Andrews JR. Effects of throwing overweight and underweight baseballs on throwing velocity and accuracy. Sports Med. 2000; 29:259-272.

14. Pavlovich L. Weighted ball training started years ago. Collegiate Baseball. 2014; 4:8.

15. Fleisig GS, Kingsley DS, Loftice JW, Dinnen KP, Ranganathan R, Dun S, et al. Kinetic comparison among the fastball, curveball, change-up, and slider in collegiate baseball pitchers. Am J Sports Med. 2006; 34:423-430.

16. Yang WW, Liu YC, Lu LC, Chang HY, Chou PP, Liu C. Performance enhancement among adolescent players after 10 weeks of pitching training with appropriate baseball weights. J Strength Cond Res.2013; 27:32453251.

17. DeRenne C, Tracy R, Dunn-Rankin P. Increasing throwing velocity. Athletic Journal. 1985; 65:36-39.

18. DeRenne C, Ho K, Blitzblau A. Effect of weighted implement training on throwing velocity. J Appl Sport Sci Res. 1990; 4:16-19.

19. Fleisig GS, Diffendaffer AX, Aune KT, Ivey B, Laughlin WA. Biomechanics analysis of weighted-ball exercise for baseball pitchers. Sports Health. 2017; 9:210-215.

20. Chiang KD, Liu C, Kao YC. Effects of throwing different weight baseballs on pitching movement and velocity. Chin J Sports Biomech. 2010; 2:22-30.

21. Shin YA, Choi WH. Effects of weighted baseball throwing 
during warm-up on ball velocity and upper extremity muscle activation in baseball pitchers. J Exerc Rehabil. 2018; 14:436-444.

22. Meister K. Injuries to the shoulder in the throwing athlete. Part one:biomechanics/ pathophysiology/classification of injury. Am J Sports Med. 2000; 28:265-275.

23. DiGiovine NM, Jobe FW, Pink M, Perry J. An electromyographic analysis of the upper extremity in pitching. J Shoulder Elbow Surg. 1992; 1:15-25.

24. US Department of Health and Human Service. Selected topics in surface electromyography for use in the occupational setting: expert perspectives. DHHSN(NIOSH) Publication, 1992, p2-5.

25. Cram JR, Kasman GS, Holtz J. Introduction to Surface Electromyography. Aspen Publication, 1998, p257-349. 26. Stone AV, Mannava S, Patel A, Marquez-Lara A, Freehill MT. Defining the Long-Toss: A Professional Baseball Epidemiological Study. Orthop J Sports Med. 2017; 5(2):2325967116686773.

27. Marsh JA, Wagshol MI, Boddy KJ, O'Connell ME, Briend SJ, Lindley KE, Caravan A. Effects of a sixweek weighted-implement throwing program on baseball pitching velocity, kinematics, arm stress, and arm range of motion. Peer J. 2018; 6: e6003.

28. Kim BG, Yoon WY. The effect of game participation by a professional pitcher on isokinetic and isometric muscle. INDJSRT. 2015; 7:123-132.

29. Reinold MM, Macrina LC, Fleisig GS, Aune K, Andrews JR. Effect of a 6-week weighted baseball throwing program on pitch velocity, pitching arm biomechanics, passive range of motion, and injury rates.Sports Health. 2018; 10:327-333.

30. Erickson BJ, Cvetanovich GL, Frank RM, Bach BR Jr, Cohen MS, Bush-Joseph CA, et al. Do clinical results and return-to-sport rates after ulnar collateral ligament reconstruction differ based on graft choice and surgical technique? Orthop J Sports Med. 2016; 4:2325967116670142.

31. Erickson BJ, Nwachukwu BU, Rosas S, Schairer WW, McCormick FM, Bach BR Jr, et al. Trends in medial ulnar collateral ligament reconstruction in the United
States: A retrospective review of a large private-payer database from 2007 to 2011. Am J Sports Med. 2015; 43:1770-1774.

32. Lipinski CL, Donvan L, McLoughlin TJ, Armstrong CW, Norte GW. Surface electromyography of the forearm musculature during an overhead throwing rehabilitation progression program. Phys Ther Sport. 2018; 33: 109116.

33. Chen FS, Rokito AS, Jobe FW. Medial elbow problems in the overhead throwing athlete. J Am Acad Orthop Surg. 2001; 9:99-113.

34. Hang YS, Lippert FG III, Spolek GA, Frankel VH, Harrington RM. Biomechanical study of the pitching elbow. Int Orthop. 1979; 3:217-223.

35. Bradley JP, Tibone JE. Electromyographic analysis of muscle action about the shoulder. Clin Sports Med. 1991; 10:789-805.

36. Jobe FW, Tibone JE, Perry J, Moynes D. An EMG analysis of the shoulder in throwing and pitching: A preliminary report. Am J Sports Med. 1983; 11:3-5.

37. Jobe FW, Moynes DR, Tibone JE, Perry J. An EMG analysis of the shoulder in pitching: A second report. Am J Sports Med. 1984; 12:218-220.

38. Kronberg M, Nemeth G, Broström LA. Muscle activity and coordination in the normal shoulder. An electromyographic study. Clin Orthop Relat Res. 1990; 257:76-85.

39. Warner JJ, Micheli LJ, Arslanian LE, Kennedy J, Kennedy R. Patterns of flexibility, laxity, and strength in normal shoulders and shoulders with instability and impingement. Am J Sports Med. 1990; 18:366-375.

40. Fleisig G, Chu Y, Weber A, Andrews J. Variability in baseball pitching biomechanics among various levels of competition. Sports Biomech. 2009; 8:10-21.

41. Werner SL, Flesig GS, Dillman CJ, Andrews JR. Biomechanics of the elbow during baseball pitching. Int J Sports Phys Ther. 1993; 17:274-278.

42. Otoshi K, Kikuchi S, Shishido H, Konno S. The proximal origins of the flexor-pronator muscles and their role in the dynamic stabilization of the elbow joint: An anatomical study.Surg Radiol Anat. 2014; 36:289-94. 
43. Pappas AM, Zawacki RM, Sullivan TJ. Biomechanics of baseball pitching. A preliminary report. Am J Sports Med. 1985; 13:216-222.

44. Ouellette H, Labis J, Bredella M, Palmer WE, Sheah K, Torriani M. Spectrum of shoulder injuries in the baseball pitcher. Skeletal Radiol. 2008; 37:491-498.

45. Dun S, Loftice J, Fleisig GS, Kingsley D, Andrews JR. A biomechanical comparison of youth baseball pitches: Is the curveball potentially harmful? Am J Sports Med. 2008; 36:686-692.
46. Keeley DW, Wicke J, Alford K, Oliver GD. Biomechanical analysis of forearm proration and its relationship to ball movement for the two-seam and four-seam fastball pitches. J Strength Cond Res. 2010; 24:2366-2371.

47. Sabick MB, Torry MR, Lawton RL, Hawkins RJ. Valgus torque in youth baseball pitchers: A biomechanical study. J Shoulder Elbow Surg. 2004; 13:349-355. 